\title{
ACTIVIDAD INSECTICIDA, REPELENTE Y ANTIALIMENTARIA DEL POLVO Y ACEITE ESENCIAL DE FRUTOS DE Schinus molle L. PARA EL CONTROL DE Sitophilus zeamais (Motschulsky)
}

\section{INSECTICIDAL, REPELLENT AND ANTIFEEDING ACTIVITY OF POWDER AND ESSENTIAL OIL OF Schinus molle L. FRUITS AGAINST Sitophilus zeamais (Motschulsky)}

\author{
Jocelyn Arias P. ${ }^{1}$, Gonzalo Silva A. ${ }^{1 *}$, Inés Figueroa C. ${ }^{1}$, Susana Fischer G. ${ }^{1}$, Agustín Robles- \\ Bermúdez $^{2}$, J. Concepción Rodríguez-Maciel ${ }^{3}$, y Angel Lagunes-Tejeda ${ }^{3}$
}

${ }^{1}$ Universidad de Concepción, Facultad de Agronomía, Departamento de Producción Vegetal, Vicente Méndez 595, Casilla 537, Chillán, Chile.

${ }^{2}$ Universidad Autónoma de Nayarit, Unidad Académica de Agricultura, Km. 9 Carr. Fed. TepicCompostela, Xalisco, Nayarit, México.

${ }^{3}$ Colegio de Postgraduados, Programa de Entomología y Acarología, Km 36,5 Carretera Federal. México-Texcoco, Montecillo, Estado de México, México.

* Autor para correspondencia: gosilva@udec.cl

\section{RESUMEN}

El pimiento boliviano (Schinus molle L.) es un árbol del cual se han identificado metabolitos secundarios con actividad insecticida. El objetivo fue evaluar, en condiciones de laboratorio, la actividad insecticida e insectistática del polvo y aceite esencial de frutos de S. molle sobre Sitophilus zeamais. En los bioensayos de toxicidad por contacto la mayor mortalidad se obtuvo con el aceite esencial al $8,0 \%$ con $96,3 \%$ de insectos muertos con una $\mathrm{CL}_{50}$ de $3,82 \mathrm{~mL}$ aceite esencial $100 \mathrm{~g}^{-1}$ maíz $(3,82 \%)$. A la misma concentración el polvo mostró un $63,8 \%$ de mortalidad y una $\mathrm{CL}_{50}$ de $7,95 \mathrm{~g}$ polvo $100 \mathrm{~g}^{-1}$ maíz $(7,95 \%)$. Ambos tratamientos no afectaron significativamente la germinación ni la pérdida de peso del grano. El aceite esencial en la concentración de 8,0\% provocó una completa inhibición en la emergencia mostrando una $\mathrm{F}_{1}$ de $0 \%$ de insectos adultos. Todos los tratamientos fueron repelentes y el mayor efecto antialimentario del aceite esencial se registró con la concentración de $16 \%$ con $71,2 \%$ de inhibición. Se concluyó que el polvo y aceite esencial de $S$. molle presentan toxicidad insecticida por contacto y efecto repelente e inhibidor de la alimentación sobre S. zeamais.

Palabras clave: pimiento boliviano, gorgojo del maíz, monoterpenos

\section{ABSTRACT}

The pepper tree (Schinus molle L.) has secondary metabolites with insecticidal activity. The objective of this study was to assess under laboratory conditions the insecticidal and insectistatic activity of fruit powder and essential oil of S. molle against Sitophilus zeamais. In contact toxicity bioassays, the highest mortality was observed with essential oil at a concentration of $8.0 \%$, recording $96.3 \%$ of dead insects with an $\mathrm{LC}_{50}$ of $3.82 \mathrm{~mL}$ essential oil $100 \mathrm{~g}^{-1}$ maize $(3.82 \%)$. Powder at the same concentration showed a $63.8 \%$ of mortality with an $\mathrm{LC}_{50}$ of $7.95 \mathrm{~g}$ powder $100 \mathrm{~g}^{-1}$ maize $(7.95 \%)$. None of the treatments affected germination or grain weight loss. The essential oil at a concentration of $8.0 \%$ caused a complete inhibition of $\mathrm{F}_{1}$ resulting in $0 \%$ of adult insects. All treatments had repellent effects. The highest antifeeding effect observed with the essential oil was recorded at a concentration 
of $16.0 \%$ with a $72.2 \%$ of inhibition. It was concluded that both powder and essential oil of $S$. molle show contact insecticidal toxicity as well as repellent and antifeeding effects against S. zeamais.

Key words: pepper tree, maize weevil, monoterpenoids

\section{INTRODUCCIÓN}

La producción de cereales, a nivel mundial, constituye una parte fundamental de la alimentación humana y animal, por lo que las pérdidas ocasionadas durante el proceso de almacenaje es un aspecto importante a considerar (Tefera, 2011).

La presencia de insectos asociados a granos almacenados trae como consecuencia la pérdida de la calidad del grano tanto para consumo como para semilla (Silva et al., 2005). Los insectos plaga causan pérdidas superiores al $10 \%$ durante la producción y de 10 a $20 \%$ en postcosecha, alcanzando en el caso de los pequeños agricultores mermas que alcanzan hasta el 40\% (García-Lara y Bergvinson, 2007).

El gorgojo del maíz (Sitophilus zeamais Motschulsky; Coleoptera: Curculionidae) es considerado mundialmente como plaga primaria de los granos almacenados pudiendo infestar las mazorcas incluso antes de que el maíz sea cosechado (García-Lara et al., 2007). Los daños a la semilla lo ocasionan tanto larvas como adultos, ya sea para alimentación u oviposición, aunque el daño más significativo lo producen los estados inmaduros que forman surcos en el endospermo al alimentarse (García-Lara y Bergvinson, 2007). Para disminuir el ataque de insectos antes o después de la cosecha, normalmente se utilizan insecticidas sintéticos, pero el mal uso de estos conduce con frecuencia a problemas como resistencia en los insectos, contaminación del ambiente y presencia de residuos en alimentos (Silva et al., 2003). A causa de lo anterior se hace necesario desarrollar técnicas que permitan reducir el uso de compuestos químicos de alta toxicidad e innovar con otras alternativas que presenten la misma eficacia pero sin los efectos negativos. Una de estas alternativas pueden ser los insecticidas de origen vegetal, que se han utilizado desde muy antigua data, como polvos, extractos o aceites esenciales (Isman et al., 2008).

El pimiento boliviano (Schinus molle L.; Anacardiaceae) es un árbol originario de la región andina de Sudamérica, principalmente Perú, aunque se extiende a Ecuador, Chile y Bolivia. Crece en suelos áridos y salinos y es muy resistente a altas temperaturas y sequía. Alcanza hasta $25 \mathrm{~m}$ de altura, de copa densa, con un tronco de hasta $1,5 \mathrm{~m}$ de diámetro y muy ramificado en la zona superior. Presenta hojas persistentes, compuestas, alternas, aromáticas, y el fruto es una drupa globosa, de 4-6 mm de diámetro, de color rosado a rojiza y brillante (Rodríguez et al., 2005). De este árbol se han extraído aceites esenciales presentes en hojas, corteza y fruto, constituidos mayoritariamente por terpenoides, a los que se les atribuye actividad insecticida (Chirino et al., 2001). Específicamente Wimalaratne et al. (1996), señalan que la actividad insecticida del aceite esencial de esta planta se debe fundamentalmente a dos compuestos: el cis-menth-2-en-l-ol y el tras-piperitol, los cuales se encuentran en una concentración del $2 \%$ en el aceite esencial de hojas y en $5 \%$ en el de frutos, además de presentar $\alpha$-pineno, $\beta$-pineno, piperina, $(+)$-limoneno, piperitona, carvacrol, mirceno, $\beta$-espatuleno y $\beta$-felandreno, entre otros.

El modo de acción de los aceites esenciales aún no se ha identificado completamente pero la sintomatología presentada por los insectos intoxicados sugiere un efecto neurotóxico (Tripathi et al., 2009). Según Isman et al. (2007), algunos constituyentes comunes de los aceites esenciales como eugenol o timol bloquean los receptores de la octopamina que es un neurotransmisor que presentan los artrópodos. Aunque estos mismos autores señalan que mecanismos físicos como disrupción de la membrana celular o bloqueo del sistema traqueal también podrían estar involucrados.

En la literatura existen antecedentes sobre la actividad insecticida de S. molle. Chirino et al. (2001) con un extracto en hexano obtuvieron un $59 \%$ de mortalidad de larvas neonatas de Cydia pomonella L. (Lepidoptera: Tortricidae). Además, este extracto también presentó efecto regulador del crecimiento afectando el paso de pupa a adulto, produciendo insectos con malformaciones. En Tribolium castaneum Herbst. (Coleoptera: Tenebrionidae), extractos etanólicos de hojas y frutos presentaron un efecto fagodisuasivo de 41 y $53 \%$ a las concentraciones de 0,7 y $6 \mathrm{mg} \mathrm{disco}^{-1}$, respectivamente (Descamps et al., 2008). El aceite esencial ha mostrado actividad insecticida por contacto, fumigante y repelente contra ninfas II de Nezara viridula L. (Hemiptera: Pentatomidae) (Werdin et al., 2008) mientras que contra Sitophilus oryzae L. (Coleoptera: Curculionidae) registra un efecto antialimentario de 62,0\% (Benzi et al., 2009). En base a lo anterior, la presente investigación tuvo como objetivo evaluar, en condiciones de laboratorio, el efecto insecticida e 
insectistático del polvo y aceite esencial de frutos de S. molle para el control de S. zeamais.

\section{MATERIALES Y MÉTODOS}

La investigación se llevó a cabo en el Laboratorio de Entomología de la Facultad de Agronomía de la Universidad de Concepción, Campus Chillán, Región del Biobío, Chile.

\section{Material vegetal}

Los frutos de $S$. molle se recolectaron en parques de la ciudad de Rancagua (34ำ10'02" S; 7043'37" W), Región del Libertador Bernardo O'Higgins, Chile, y se trasladaron al Laboratorio de Entomología donde se secaron en una estufa de convección forzada (Mermet Gmbh, UNB 500, Schwabach, Germany) a $40 \pm 1^{\circ} \mathrm{C}$ por $72 \mathrm{~h}$.

El polvo se obtuvo moliendo los frutos, primero con un molino manual y luego en un molino eléctrico para café (Moulinex, A5052HF, Aleçon, France). En seguida, el polvo obtenido se tamizó con un tamiz de 18 mesh (Dual Manufacturing, Chicago, Illinois, USA) para homogenizar la granulometría. Este procedimiento se realizó el mismo día en que se montaron los bioensayos.

$\mathrm{El}$ aceite esencial se extrajo a partir de $200 \mathrm{~g}$ de polvo y $500 \mathrm{~mL}$ de agua destilada mediante destilación por arrastre de vapor con un aparato tipo Clevenger durante $2 \mathrm{~h}$ (Vogel et al., 1997). Luego, el aceite obtenido se dejó en un congelador a $-2,5 \pm 1^{\circ} \mathrm{C}$, para separar el agua del aceite por diferencia de fases, y se almacenó en un frasco ámbar conservado en frío $\left(4,5 \pm 2^{\circ} \mathrm{C}\right)$ hasta su utilización en los bioensayos.

El análisis fitoquímico de los principales constituyentes del aceite esencial de S. molle se realizó de acuerdo a Adams (2007) en un cromatográfo de gases acoplado a un espectométro de masa (HPGC-MS; HP 5890 Series II, Hewlett Packard, Palo Alto, California, USA) en el Laboratorio de Farmacognosia del Departamento de Química y Farmacia perteneciente a la Facultad de Farmacia de la Universidad de Concepción.

\section{Insectos y cereal}

Se utilizaron adultos de S. zeamais los que se obtuvieron de la colonia permanente del Laboratorio de Entomología, y para obtener la cantidad necesaria de insectos adultos se reprodujeron en una cámara bioclimática (Mermet Gmbh, UNB 800, Schwabach, Germany) a una temperatura de $25 \pm 2^{\circ} \mathrm{C}, 60 \% \mathrm{HR}$ y completa oscuridad. Se utilizó maíz (Zea mais L.) cv. Dekalb DK440 como sustrato alimenticio, el mismo que posteriormente se empleó en los bioensayos.
El maíz se obtuvo de la Estación Experimental "El Nogal" de la Facultad de Agronomía de la Universidad de Concepción en Chillán; se lavó con agua potable y luego se refrigeró por $48 \mathrm{~h}$ a una temperatura de $2,5 \pm 1^{\circ} \mathrm{C}$, para eliminar alguna infestación natural con insectos o residuo de insecticida que pudiera afectar los resultados de los bioensayos.

\section{Toxicidad por contacto}

En este bioensayo se utilizó la metodología de Tavares y Vendramim (2005) con ligeras modificaciones. En frascos de vidrio de 250 $\mathrm{mL}$ se colocaron $100 \mathrm{~g}$ de maíz mezclado con el polvo o aceite esencial de acuerdo a los respectivos tratamientos: $0 ; 0,5 ; 1,0 ; 2,0 ; 4,0$ y $8,0 \%$. En el caso de los bioensayos con aceite esencial este se diluyó previamente en $1 \mathrm{~mL}$ de acetona para posteriormente ser mezclado con el maíz y dejado a temperatura ambiente $(25 \pm$ $2^{\circ} \mathrm{C}$ ) durante $1 \mathrm{~h}$ para que la acetona se evaporara (Obeng-Ofori and Reichmuth, 1997). Luego, los frascos se infestaron con 10 parejas de insectos de una semana de edad diferenciados por sexo con el criterio de Halstead (1963). Cada unidad experimental se etiquetó con la información del respectivo tratamiento y se almacenó en una cámara bioclimática en las mismas condiciones indicadas para la cría masiva de insectos. Los tratamientos tuvieron cuatro repeticiones incluyendo un testigo formado por el grano infestado con las 10 parejas de insectos en el caso del polvo y maíz más $1 \mathrm{~mL}$ de acetona y 10 parejas de insectos en el aceite esencial.

A los 15 días después de la infestación (DDI) se cuantificaron los insectos adultos vivos y muertos de cada tratamiento y se calculó el porcentaje de mortalidad que se corrigió con la fórmula de Abbott (1925). Una vez registrada la mortalidad y retirados todos los insectos, los frascos se devolvieron a la cámara bioclimática por 40 días más (55 DDI) para, al final de este período, evaluar la emergencia de insectos adultos $\left(\mathrm{F}_{1}\right)$ y la pérdida de peso del grano. En el caso de la $\mathrm{F}_{1}$ el testigo se consideró como $100 \%$.

Finalmente, también a los 55 DDI se evaluó si el polvo o aceite esencial afectaron la germinación de las semillas utilizando la metodología de Pérez et al. (2007). Para ello se eligieron por repetición 30 semillas sin daño aparente y se colocaron a germinar en placas Petri de vidrio de $9 \mathrm{~cm}$ de diámetro (Normax, Marinha Grande, Portugal) acondicionadas con papel filtro húmedo durante 7 días a temperatura ambiente $\left(25 \pm 2^{\circ} \mathrm{C}\right)$. $\mathrm{Al}$ cabo de este periodo se evaluó el porcentaje de germinación considerando como $100 \%$ al número de semillas germinadas en el testigo. 


\section{Repelencia}

En este bioensayo se utilizó la metodología de Tavares y Vendramim (2005), que consiste en una arena de libre elección, formada por cinco placas Petri plásticas (Miniplast Ein-Shemer ACS Ltd., Kibbutz Ein Shemer, Post Heffer, Israel) de $5 \mathrm{~cm}$ de diámetro, donde una placa central está conectada a las cuatro restantes por tubos plásticos de $10 \mathrm{~cm}$ de longitud formando una " $X$ ". Cada placa tenía 20 g de maíz y los tratamientos (polvo o aceite esencial) al igual que el testigo se distribuyeron en dos placas simétricamente opuestas. En el caso del polvo éste se mezcló directamente con el grano, mientras que el aceite esencial se diluyó en $1 \mathrm{~mL}$ de acetona, luego la solución se mezcló con el grano y se esperó durante $1 \mathrm{~h}$ para permitir la volatilización del solvente. A continuación, en la placa central se liberaron 20 insectos adultos sin diferenciación de sexos y después de $24 \mathrm{~h}$ se contabilizó el número de insectos por placa. Con los datos obtenidos se calculó el índice de repelencia (IR) de Mazzonetto y Vendramim (2003) que clasifica al tratamiento como neutro si $\mathrm{IR}=1$, atrayente si IR $>1$ y repelente $\mathrm{si}$ IR $<1$. Las concentraciones evaluadas para el polvo y aceite esencial fueron 0,$5 ; 1,0 ; 2,0 ; 4,0$ y $8,0 \%$ teniendo cada tratamiento cuatro repeticiones.

\section{Efecto antialimentario}

La evaluación de la actividad antialimentaria se realizó solamente para el aceite esencial, utilizando el método de cubos de harina sin posibilidad de elección (Huang et al., 2002). Se mezclaron $100 \mathrm{~mL}$ de agua destilada con $40 \mathrm{~g}$ de harina de trigo, hasta que se obtuvo una solución homogénea. A continuación se colocaron $3 \mathrm{~mL}$ de esta solución en cada alveolo de una cubetera de plástico para hielo y se dejaron secar en un horno de convección forzada por $24 \mathrm{~h}$ a $60 \pm 1^{\circ} \mathrm{C}$ de temperatura. En seguida, en tubos Eppendorf se preparó la solución de aceite esencial en acetona en concentraciones de 0,$5 ; 1,0 ; 2,0 ; 4,0 ; 8,0$ y $16 \%$ $(\mathrm{v} / \mathrm{v})$, más un testigo consistente en solo acetona. Una vez secos los cubos se impregnaron con 20 $\mu \mathrm{L}$ de la solución de aceite esencial y se registró el peso inicial. Luego, los cubos se colocaron individualmente en placas petri plásticas de 5 $\mathrm{cm}$ de diámetro, las que se infestaron con cinco insectos adultos que llevaban $24 \mathrm{~h}$ sin alimentarse. Se realizaron cuatro repeticiones por tratamiento y a las $72 \mathrm{~h}$ de iniciado el bioensayo se registró nuevamente el peso de cada cubo de harina, descontándose una posible pérdida o ganancia de humedad con base en cubos de referencia tratados con $20 \mu \mathrm{L}$ de acetona pero sin infestar con insectos que se dejaron al inicio del bioensayo en las mismas condiciones que los tratamientos. Con estos datos se calculó la actividad antialimentaria del tratamiento utilizando la fórmula de Farrar et al. (1989):

Índice Antialimentario $(\mathrm{IA})=\left[(\mathrm{C}-\mathrm{T}) \times \mathrm{C}^{-1}\right] \times 100$, dónde: $\mathrm{C}$ : consumo de los cubos en el control (mg); T: consumo de los cubos tratados (mg).

\section{Análisis estadístico y diseño experimental}

El diseño experimental fue completamente al azar. Los datos obtenidos en cada bioensayo se analizaron con una prueba de normalidad Shapiro-Wilks modificado y de homogeneidad de varianzas de Levene para comprobar los supuestos del análisis de varianza (ANDEVA). Los datos que no cumplieron con los supuestos se transformaron con la fórmula arcoseno $(\mathrm{x} / 100)^{0,5}$ y se sometieron a un análisis de varianza y a un test de comparación de medias con la prueba de Tukey con una significancia de $5 \%(\alpha=0,05)$. Las concentraciones letal $50 \%\left(\mathrm{CL}_{50}\right)$ y $90 \%\left(\mathrm{CL}_{90}\right)$ se obtuvieron con un análisis Probit (Finney, 1971). Ambos análisis se realizaron con el software Statistical Analysis System (SAS).

\section{RESULTADOS Y DISCUSIÓN}

\section{Análisis fitoquímico}

El análisis fitoquímico realizado al aceite esencial de S. molle identificó un $71,8 \%$ de los componentes indicando que estos mayoritariamente pertenecen al grupo de los terpenos (Tabla 1). Los que se encontraron en mayor proporción fueron $\beta$-terpineno (6,6\%), $\beta$-pineno (15,4\%), $\alpha$-felandreno $(14,9 \%)$ y $\mathrm{Q}$-cimeno $(10,8 \%)$. Estos resultados coinciden con Murray et al. (2005), quienes en tres especies del género Schinus también identificaron a $\alpha$-felandreno y $\beta$-pineno como los más abundantes.

\section{Toxicidad por contacto}

La mayor mortalidad del polvo de frutos de $S$. molle se obtuvo con la concentración de $8,0 \%$, con un $63,8 \%$ de insectos muertos (Tabla 2). Este resultado según Lagunes (1993) clasifica al polvo de $S$. molle como prometedor, debido a que superó el $50 \%$ de mortalidad. En el caso de las concentraciones restantes la mortalidad no sobrepaso el $30 \%$, y no presentaron diferencias significativas entre ellas. $\mathrm{La} \mathrm{CL}_{50}$ y $\mathrm{CL}_{90}$ obtenidas fueron de 7,95 y 24,3\% (equivalentes a 79,3 y $243 \mathrm{~g}$ polvo $\mathrm{kg}^{-1}$ grano) respectivamente. La actividad insecticida del polvo de $S$. molle se puede deber a la presencia de monoterpenos los cuales son liberados al momento de moler los frutos. Aunque, según Awoke et al. (2014) las hojas también presentarían compuestos con propiedades insecticidas ya que estos autores reportan sobre un $80 \%$ de mortalidad de $S$. zeamais con concentraciones iguales o superiores 
Arias P., J. Actividad insecticida, repelente y antialimentaria Schinus molle L. para el control de 97 Sitophilus zeamais

Tabla 1. Análisis fitoquímico del aceite esencial de frutos de Schinus molle

Table 1. Chemical analysis of essential oil of Schinus molle fruits.

\begin{tabular}{|c|c|c|}
\hline Compuesto & $\begin{array}{c}\text { Abundancia } \\
(\%)\end{array}$ & Identificación $^{1}$ \\
\hline b-terpineno & 6,63 & $\mathrm{IK}, \mathrm{MS}$ \\
\hline Isoterpinoleno & 0,16 & IK, MS \\
\hline b-pineno & 15,40 & $\mathrm{IK}, \mathrm{MS}$ \\
\hline a-felandreno & 14,98 & $\mathrm{IK}, \mathrm{MS}$ \\
\hline p-cimeno & 10,87 & $\mathrm{IK}, \mathrm{MS}$ \\
\hline 1-8-cineol & 0,24 & $\mathrm{IK}, \mathrm{MS}$ \\
\hline Ácido octanoico metil ester & 1,38 & $\mathrm{IK}, \mathrm{MS}$ \\
\hline 1,3-dimetil-1-ciclohexeno & 0,27 & $\mathrm{IK}, \mathrm{MS}$ \\
\hline (-)-terpinen-4-ol & 0,11 & $\mathrm{IK}, \mathrm{MS}$ \\
\hline Carvenona & 0,13 & $\mathrm{IK}, \mathrm{MS}$ \\
\hline 4-isopropil-2-ciclohexanona & 0,39 & $\mathrm{IK}, \mathrm{MS}$ \\
\hline Sabinil acetato & 0,61 & $\mathrm{IK}, \mathrm{MS}$ \\
\hline 8-oxabiciclo[5.1.0]oct-5-en-2-ol,1,4,4-trimetil & 0,24 & $\mathrm{IK}, \mathrm{MS}$ \\
\hline 5,9-dimetildeca-5,8-dien-2-ona & 3,76 & $\mathrm{IK}, \mathrm{MS}$ \\
\hline 3-heptin-2-ol, 2-metil- & 0,36 & $\mathrm{IK}, \mathrm{MS}$ \\
\hline Safrol & 0,76 & IK, MS \\
\hline Limoneno glicol & 1,12 & $\mathrm{IK}, \mathrm{MS}$ \\
\hline Desconocido & 1,08 & $\mathrm{IK}, \mathrm{MS}$ \\
\hline Citronelil acetato & 0,20 & $\mathrm{IK}, \mathrm{MS}$ \\
\hline$(-/+)$-lavandulol acetato & 0,31 & $\mathrm{IK}, \mathrm{MS}$ \\
\hline (-)-germacreno A & 0,17 & $\mathrm{IK}, \mathrm{MS}$ \\
\hline Desconocido & 0,29 & $\mathrm{IK}, \mathrm{MS}$ \\
\hline Delta selineno & 0,56 & $\mathrm{IK}, \mathrm{MS}$ \\
\hline Desconocido & 0,26 & $\mathrm{IK}, \mathrm{MS}$ \\
\hline Cariofileno & 2,88 & $\mathrm{IK}, \mathrm{MS}$ \\
\hline a-cariofileno & 0,60 & $\mathrm{IK}, \mathrm{MS}$ \\
\hline b-cubebeno & 0,33 & $\mathrm{IK}, \mathrm{MS}$ \\
\hline a-muuroleno & 0,79 & $\mathrm{IK}, \mathrm{MS}$ \\
\hline Isoledeno & 0,54 & $\mathrm{IK}, \mathrm{MS}$ \\
\hline Cadineno & 2,94 & $\mathrm{IK}, \mathrm{MS}$ \\
\hline Gamma-himachaleno & 0,82 & $\mathrm{IK}, \mathrm{MS}$ \\
\hline a-cadinol & 0,73 & $\mathrm{IK}, \mathrm{MS}$ \\
\hline (-)-alloisologifoleno & 0,92 & $\mathrm{IK}, \mathrm{MS}$ \\
\hline a-santanol & 0,84 & $\mathrm{IK}, \mathrm{MS}$ \\
\hline$(1 \mathrm{R}, 5 \mathrm{R})$-carvil acetato & 0,11 & $\mathrm{IK}, \mathrm{MS}$ \\
\hline
\end{tabular}

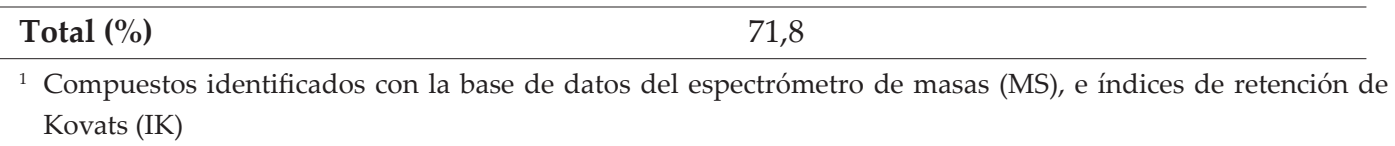

a 2,5\% de polvo de follaje de esta planta. Pero, la toxicidad obtenida en la presente investigación es superior a la reportada por Silva et al. (2005), quienes utilizando polvo de frutos de $S$. molle a una concentración de 1,0\% registraron una mortalidad de $S$. zeamais de $1,8 \%$. Sin embargo, el porcentaje de mortalidad registrado en este bioensayo, a pesar de sobrepasar el $50 \%$, es menor al reportado para otras especies vegetales evaluadas para el control de S. zeamais como polvo de canelo (Drimys winteri J. R. et G. Forster; Winteraceae) que en concentraciones de 1,$0 ; 2,0$ y 4,0\% registró una mortalidad de 85,9 y $100 \%$ respectivamente (Andrade et al., 2009), y Peumus boldus Molina (Monimiaceae) que al 1,0 y 2,0\% exhibió una mortalidad de 66 y 100\% (Bustos et al., 2009).

La mayor toxicidad del aceite esencial 
Tabla 2. Mortalidad (\%) de Sitophilus zeamais tratado con polvo y aceite esencial de frutos de Schinus molle.

Table 2. Mortality (\%) of Sitophilus zeamais treated with powder and essential oil of fruits of Schinus molle.

\begin{tabular}{ccc}
\hline Concentración & Polvo & Aceite esencial \\
\hline 0,5 & $1,3 \mathrm{~b}$ & $1,3 \mathrm{c}$ \\
1,0 & $1,3 \mathrm{~b}$ & $2,5 \mathrm{bc}$ \\
2,0 & $0,2 \mathrm{~b}$ & $1,3 \mathrm{c}$ \\
4,0 & $20 \mathrm{a}$ & $45 \mathrm{~b}$ \\
8,0 & $63,8 \mathrm{a}$ & $96,3 \mathrm{a}$ \\
\hline $\mathrm{C} . \mathrm{V} .(\%)$ & 5,12 & 54,6 \\
$\mathrm{n}^{2}$ & 80 & 80 \\
$\mathrm{~b}^{3}$ & 2,62 & 3,38 \\
$\mathrm{CL}_{50}{ }^{4}$ & 7,95 & 3,82 \\
$(95 \% \mathrm{IC})^{5}$ & $6,63-9,51$ & $3,4-4,3$ \\
$\mathrm{CL}_{90}{ }^{6}$ & 24,3 & 9,09 \\
$(95 \% \mathrm{IC})^{5}$ & $15,9-36$ & $7,3-10,8$ \\
\hline
\end{tabular}

* Tratamientos con igual letra en la columna no difieren estadísticamente, Tukey $(p>0,05)$. C.V: Coeficiente de variación.

${ }^{1}$ Datos transformados previamente al análisis de varianza con la fórmula arcoseno $(\mathrm{x} / 100)^{0,5}$

${ }^{2}$ Número de insectos tratados.

${ }^{3}$ Pendiente de la línea dosis-Probit (Ldp).

${ }^{4}$ Concentración letal 50\% (equivalente a g polvo por $100 \mathrm{~g}$ cereal y $\mathrm{mL}$ aceite esencial por $100 \mathrm{~g}$ cereal, respectivamente).

${ }^{5}$ Intervalos de Confianza (IC) al 95\% de probabilidad.

${ }^{6}$ Concentración letal $90 \%$ (equivalente a g polvo por $100 \mathrm{~g}$ cereal y $\mathrm{mL}$ aceite esencial por $100 \mathrm{~g}$ cereal respectivamente).

también se registró con la concentración de $8,0 \%$, presentando un $96,3 \%$ de mortalidad, valor significativamente mayor que las restantes concentraciones. Esta toxicidad se manifestó en una $\mathrm{CL}_{50}$ y $\mathrm{CL}_{90}$ de 3,82 y 9,1\% (equivalentes a 38,2 y $91 \mathrm{~mL}$ aceite esencial $\mathrm{kg}^{-1}$ grano) respectivamente (Tabla 2). El aceite esencial al igual que el polvo se puede clasificar como prometedor, debido a que sobrepasa el $50 \%$ de mortalidad (Lagunes, 1993).

El efecto insecticida de contacto del aceite esencial de $S$. molle coincide con lo reportado por Abdel-Sattar et al. (2010), quienes con una concentración de $1000 \mu \mathrm{L}$ en discos de papel filtro, obtuvieron una mortalidad de 90 y 93,3\% para Trogoderma granarium Everts. (Coleoptera: Dermestidae) y Tribolium castaneum Herbst. (Coleoptera: Tenebrionidae), respectivamente. Además, los resultados obtenidos también concuerdan con Werdin et al. (2008), quienes con concentraciones de 22,8 y $176 \mu \mathrm{g}$ de aceite esencial en hexano reportan un efecto insecticida de $90 \%$ sobre ninfas de segundo instar de Nezara viridula L. (Hemiptera: Pentatomidae).

La actividad insecticida del aceite esencial de $S$. molle se puede deber a la presencia de monoterpenos, compuestos a los que se le han atribuido propiedades insecticidas y antimicrobiales (Kordali et al., 2007; Hayouni et al., 2008; Isman et al., 2008). Específicamente de los compuestos identificados en el análisis fitoquímico $\beta$-pineno presenta propiedades insecticidas, ya que Asawalam et al. (2008) aislaron este metabolito del aceite esencial de Vernonia amigdalina Delile (Compositae) y Xylopia aethiopica (Dunal) A. Rich (Annonaceae) obteniendo 37 y $46 \%$ de mortalidad por contacto sobre $S$. zeamais, respectivamente. Igualmente Iannacone y Alvariño (2010) señalan que $\alpha$-felandreno, que en el aceite esencial utilizado en la presente investigación se encontró en un 14,9\%, es uno de los cuatro compuestos responsables de la actividad insecticida y repelente del aceite esencial de S. molle contra Varroa destructor Anderson \& Trueman (Mesostigmata: Varroidae).

A pesar de que tanto en el polvo como en el aceite esencial se produjo mayor mortalidad cuando se incrementó la concentración, a iguales concentraciones el polvo mostró menor toxicidad $(63,8 \%)$ que el aceite esencial (96,3\%). Esto se refrenda en el valor de la pendiente de la línea dosis probit (Ldp) (Fig. 1) en que para el polvo fue de 2,62 mientras que en el aceite esencial fue de 3,38 (Tabla 2). De acuerdo a Lagunes (1993) el 


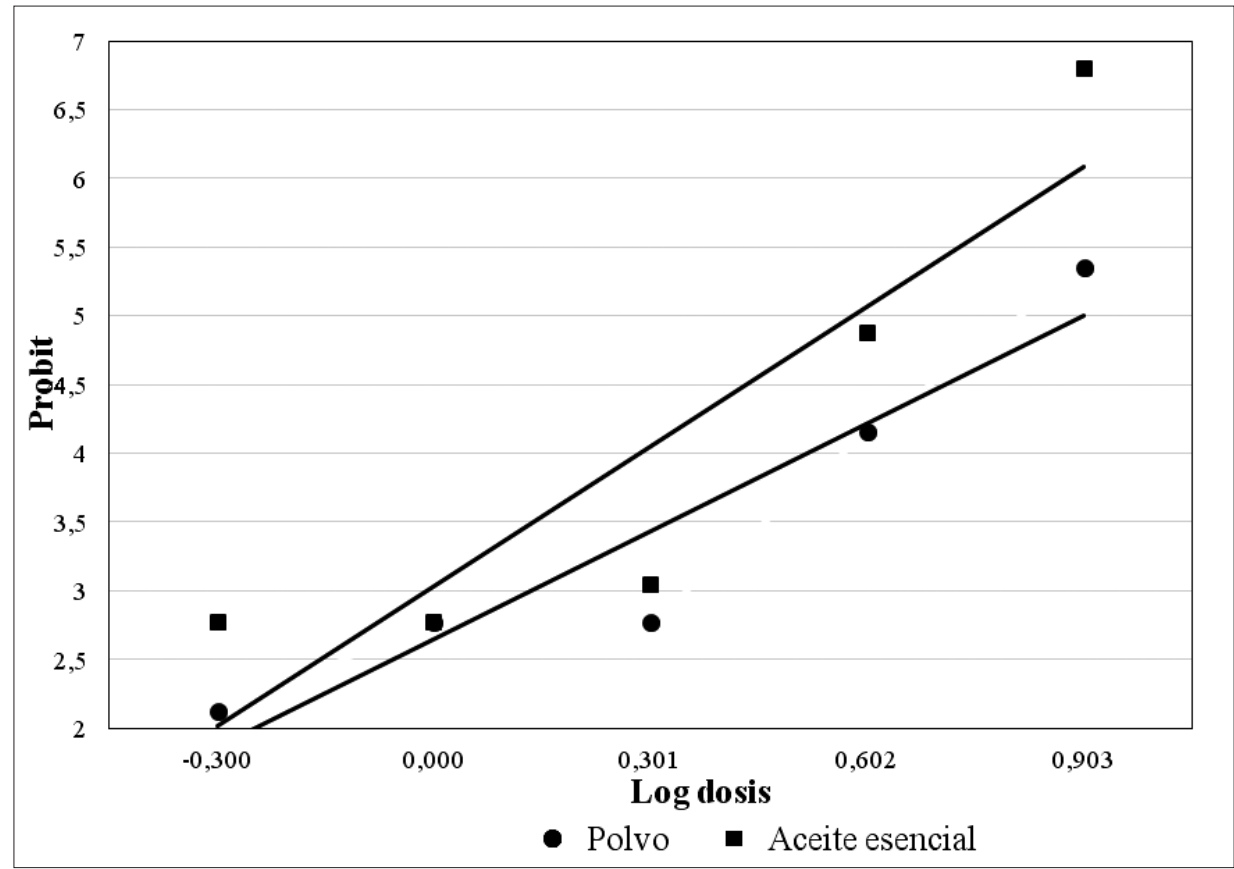

Fig. 1. Línea dosis-probit (Ldp) de la toxicidad por contacto del polvo y aceite esencial de Schinus molle sobre Sitophilus zeamais.

Fig. 1. Dose-probit line (Ldp) of contact toxicity of powder and essential oil of Schinus molle against Sitophilus zeamais.

que la pendiente de la Ldp presente un valor más alto indica una mayor toxicidad del compuesto evaluado junto con que el organismo blanco presenta una susceptibilidad genéticamente más homogénea. Aunque se debe considerar que con el polvo se obtiene mayor rendimiento del material vegetal, debido a que para obtener aceite esencial se necesita una mayor cantidad de follaje y tiempo de extracción, además de requerir equipo especializado para su obtención.

\section{Emergencia de insectos adultos $\left(\mathrm{F}_{1}\right)$}

En los tratamientos con polvo se observa una disminución significativa de la $\mathrm{F}_{1}$ especialmente con la concentración más alta $(8,0 \%)$, en la que emergió un 26,3\% (73,7\% de inhibición) de insectos adultos. Este tratamiento fue el único que presentó diferencias estadísticas con el testigo, siendo la concentración de 0,5\% la que mostró la mayor emergencia, con $84,3 \%$ (15,7\% de inhibición) (Tabla 3). Estos resultados coinciden con estudios realizados con polvo de otras especies vegetales que han reducido la emergencia de insectos adultos, como $P$. boldus que en concentraciones de 0,$5 ; 1,0$ y $2 \%$ no superó el 20\% (80\% de inhibición) (Pérez et al., 2007).

La menor $F_{1}$ de $S$. zeamais en maíz tratado con aceite esencial se alcanzó con la concentración de $8,0 \%$ con $0 \%$ ( $100 \%$ de inhibición) mientras que la emergencia de los restantes tratamientos fluctuó entre 18,5 y 30,5\%, no observándose diferencias estadísticas entre ellos, pero sí con 8,0\%.

La reducida emergencia de insectos adultos registrada en los tratamientos con aceite esencial se relaciona directamente con la mortalidad obtenida en el bioensayo de toxicidad por contacto, que en el caso de la concentración de $8 \%$ fue de $96,3 \%$ de insectos muertos. Los resultados obtenidos son similares a los de aceites esenciales de otras plantas, como Ocimum kilimandscharicum L. (Lamiaceae), que inhibió completamente la progenie de S. granarius y S. zeamais (Obeng-Ofori et al., 1998), y P. boldus que en concentraciones de 1,2 y $4 \%$ registraron un $0 \%$ de emergencia de $S$. zeamais (Betancur et al., 2010).

\section{Pérdida de peso}

La mayor pérdida de peso del maíz tratado con polvo de $S$. molle se registró con las concentraciones menores de polvo, las cuales tuvieron valores entre 4,1 y 5,2\%, sin presentar diferencias estadísticas entre ellas pero si con el testigo que fue significativamente mayor con $7,4 \%$. El tratamiento de $8,0 \%$ presentó la menor pérdida de peso con $1,4 \%$ siendo significativamente diferente a todas las restantes concentraciones (Tabla 3). Por tanto, se puede inferir que existe una relación inversa entre la pérdida de peso y la toxicidad por contacto, debido a que en las concentraciones más bajas de polvo se produjo 
Tabla 3. Emergencia de insectos adultos $\left(\mathrm{F}_{1}\right)(\%)$ de Sitophilus zeamais, pérdida de peso y germinación de maíz tratado con polvo y aceite esencial de frutos de Schinus molle.

Table 3. Emergence of adult insect $\left(\mathrm{F}_{1}\right)(\%)$ of Sitophilus zeamais, maize weight loss and germination treated with powder and essential oil of fruits of Schinus molle.

\begin{tabular}{|c|c|c|c|c|c|c|}
\hline \multirow[b]{2}{*}{ Concentración } & \multicolumn{2}{|c|}{ Emergencia $\left(F^{1}\right)^{1 *}$} & \multicolumn{2}{|c|}{ Pérdida de peso ${ }^{1 *}$} & \multicolumn{2}{|c|}{ Germinación $^{1 *}$} \\
\hline & Polvo & Aceite esencial & Polvo & Aceite esencial & Polvo & Aceite esencial \\
\hline Testigo & $100 \mathrm{~b}$ & $100 \mathrm{c}$ & $7,4 \mathrm{c}$ & $7,4 \mathrm{c}$ & $100 \mathrm{a}$ & $100 \mathrm{a}$ \\
\hline 0,5 & $84,3 \mathrm{~b}$ & $30,5 \mathrm{~b}$ & $5,2 \mathrm{~b}$ & $5,2 \mathrm{~b}$ & $89 \mathrm{~b}$ & $77 \mathrm{c}$ \\
\hline 1,0 & $71,0 \mathrm{~b}$ & $23,0 \mathrm{~b}$ & $4,6 \mathrm{~b}$ & $5,7 \mathrm{~b}$ & $89 \mathrm{~b}$ & $87 \mathrm{bc}$ \\
\hline 2,0 & $69,0 \mathrm{~b}$ & $30,8 \mathrm{~b}$ & $4,8 \mathrm{~b}$ & $3,9 \mathrm{~b}$ & $89 \mathrm{~b}$ & 81 bc \\
\hline 4,0 & $64,3 \mathrm{~b}$ & $18,5 \mathrm{~b}$ & $4,1 \mathrm{~b}$ & $3,1 \mathrm{~b}$ & $93 \mathrm{ab}$ & $88 \mathrm{~b}$ \\
\hline 8,0 & $26,3 \mathrm{a}$ & $0,0 \mathrm{a}$ & $1,4 \mathrm{a}$ & $0,1 \mathrm{a}$ & $93 \mathrm{ab}$ & $97 \mathrm{a}$ \\
\hline C.V. (\%) & 12,9 & 27,7 & 6,56 & 19,8 & 7,6 & 5,5 \\
\hline
\end{tabular}

* Tratamientos con igual letra en la columna no difieren estadísticamente, Tukey ( $p>0,05)$.

C.V: Coeficiente de variación.

${ }^{1}$ Datos transformados previamente al análisis de varianza con la fórmula arcoseno $(\mathrm{x} / 100)^{0,5}$

una menor mortalidad, lo que implica una mayor cantidad de insectos alimentándose y por ende mayor efecto sobre el peso del grano. Esto se corrobora con la concentración de 8,0\%, que registró la mayor mortalidad y a la vez la menor pérdida de peso del grano. Respecto a esta variable existen antecedentes que ratifican la tendencia de que a menor concentración de polvo mayor es la pérdida de peso del grano, como Andrade et al. (2009) con polvo de D. winteri, Silva et al. (2005) con C. ambrosoides y P. boldus, y Salvadores et al. (2007) para polvo de Capsicum annuum var longum (DC.) Sendth (Solanaceae), Cinnamomum zeylanicum Blume (Laureaceae) y Myristica fragans Houtt. (Myristicaceae).

En las pruebas con aceite esencial la menor pérdida de peso fue de 0,1\% y también se observó en la mayor concentración evaluada $(8,0 \%)$, tratamiento que difirió estadísticamente de las concentraciones menores (Tabla 3). Los restantes tratamientos tuvieron una pérdida de peso entre 3,1 y $7,4 \%$ y se observó la misma tendencia que para el polvo de $S$. molle de una mayor concentración asociada a una menor pérdida de peso.

\section{Germinación}

En los bioensayos realizados tanto para polvo como aceite esencial no se observó una pérdida significativa en el poder germinativo de las semillas de maíz.

Los tratamientos con polvo de S. molle no presentaron diferencias estadísticas entre ellos, mostrado una germinación entre 89 y 93\% (Tabla 3). Estos resultados son superiores a los de otras especies como P. boldus, cuyo polvo según
Pérez et al. (2007) en la medida que aumenta su concentración disminuye la germinación del maíz. Además aunque no se utilizó semilla certificada, la germinación obtenida en los tratamientos de 4,0 y $8,0 \%$ se encuentra por sobre el $90 \%$ que exige el Servicio Agrícola y Ganadero (SAG) para que el maíz sea comercializado como semilla (SAG, 2000) por lo que estos tratamientos se podrían utilizar para proteger grano destinado tanto para alimentación como para semilla.

El aceite esencial de S. molle tampoco afectó significativamente la germinación del maíz. Los tratamientos entre 0,5 y $4,0 \%$ no mostraron diferencias estadísticas entre sí con valores de germinación entre 77 y $88 \%$ (Tabla 3). La germinación obtenida en la concentración de $8,0 \%(97 \%)$, fue significativamente mayor que los demás tratamientos de aceite esencial, lo que podría deberse a que como este tratamiento presentó la menor emergencia de insectos adultos $\left(\mathrm{F}_{1}\right)$ hubo un menor número de insectos que se alimentaron, produciendo un mayor porcentaje de granos sanos que no perdieron su poder germinativo.

\section{Repelencia}

En los bioensayos realizados con polvo todos los tratamientos registraron valores de IR menores a 1, lo que según Mazzoneto y Vendramim (2003) significa que poseen efecto repelente sobre adultos de S. zeamais (Tabla 3). Sin embargo, los valores del IR más cercanos a 0 se observan con las concentraciones más altas, lo cual implica un mayor efecto repelente. Con respecto a este bioensayo, los resultados son superiores a los de Awoke et al. (2014) quienes requirieron de 
Arias P., J. Actividad insecticida, repelente y antialimentaria Schinus molle L. para el control de

concentraciones de polvo de S. molle iguales o superiores a $2,5 \%$ para obtener repelencia sobre adultos de $S$. zeamais.

El aceite esencial también mostró en todos los tratamientos valores de IR menores a 1 , fluctuando este índice entre 0,2 y 0,7 (Tabla 4). Los valores concuerdan con Fernandes y Favero (2014), quienes reportan acción repelente del aceite esencial de $S$. molle contra $S$. zeamais a partir de las $48 \mathrm{~h}$. Werdin et al. (2008) obtuvieron resultados similares con aceite esencial de $S$. molle a una concentración de $5,2 \mathrm{mg} \mathrm{mL}^{-1}$ sobre $N$. viridula. Sin embargo, coinciden parcialmente con Benzi et al. (2009), quienes con concentraciones de 0,04 y $0,4 \%$ de aceite esencial de follaje y frutos de $S$. molle reportaron un mayor efecto de repelencia sobre $S$. oryzae con el follaje que con los frutos. Específicamente estos autores obtuvieron repelencia con la concentración de $0,04 \%$ de aceite esencial de frutos y no con la de $0,4 \%$ resultando incluso, a pesar de su mayor concentración, este último tratamiento con efecto atrayente.

Según Maganga et al. (1996) la repelencia de $S$. molle podría deberse a la presencia de Q-cimeno, compuesto que ha mostrado esta propiedad sobre Musca domestica L. (Diptera: Muscidae), y considerando que el análisis fitoquímico indica que este compuesto se encuentra en un $14,9 \%$ en el aceite esencial de $S$. molle utilizado en la presente investigación este efecto podría atribuirse a su presencia.

Finalmente, se observó que en todos los bioensayos (polvo y aceite esencial) existe una relación directa entre la concentración utilizada y la repelencia, es decir, a mayor concentración mayor es el efecto repelente. Esta tendencia también fue reportada por Fernandes y Favero (2014), y según Nerio et al. (2009) y Conti et al. (2010), esto se explica en que a mayor concentración de polvo o aceite esencial mayor es también la concentración de terpenos, por lo que es lógico obtener un incremento en la actividad repelente al aumentar la dosis.

\section{Efecto antialimentario}

El máximo efecto antialimentario del aceite esencial de $S$. molle se logró con la concentración de $16 \%$ con un IA de $71,2 \%$, aunque sin diferencias significativas con los tratamientos entre 1 y $8 \%$, que presentaron una inhibición de la alimentación entre 37,8 y 53,4\%, respectivamente. Se observaron diferencias estadísticas solamente entre las concentraciones de 0,5 y $16 \%$ (la menor y mayor concentración evaluada, respectivamente) presentando estos tratamientos un IA de 27,8 y $71,2 \%$, respectivamente (Tabla 5). La inhibición en la alimentación de adultos de $S$. zeamais registrada en estos bioensayos concuerda con Benzi et al. (2009), quienes obtuvieron un efecto antialimentario de $62,0 \%$ sobre $S$. oryzae utilizando aceite esencial de $S$. molle a una concentración de $4 \mathrm{mg}$ disco $^{-1}$.

Finalmente, en base a los bioensayos realizados en la presente investigación se puede señalar que $S$. molle es una especie vegetal prometedora para el control de S. zeamais, pero que debe ser validada con bioensayos en bodega.

\section{CONCLUSIÓN}

Los resultados de la presente investigación permiten concluir que el polvo y aceite esencial de frutos de $S$. molle presentan efecto insecticida de contacto junto con actividad antialimentaria y repelente sobre adultos de $S$. zeamais. Además, estos se podrían utilizar para proteger cereales destinados tanto para alimentación animal como semilla ya que no afectan la germinación del maíz.

Tabla 4. Efecto repelente del polvo y aceite esencial de frutos de Schinus molle sobre Sitophilus zeamais.

Table 4. Repellent effect of powder and essential oil of Schinus molle fruits against Sitophilus zeamais.

\begin{tabular}{ccc}
\cline { 2 - 3 } Concentración, \% & \multicolumn{2}{c}{ Índice de Repelencia (IR) } \\
\cline { 2 - 3 } & Polvo & Aceite esencial \\
\hline 0,5 & $0,2 \mathrm{a}$ & $0,7 \mathrm{a}$ \\
1,0 & $0,2 \mathrm{a}$ & $0,6 \mathrm{a}$ \\
2,0 & $0,1 \mathrm{a}$ & $0,6 \mathrm{a}$ \\
4,0 & $0,0 \mathrm{a}$ & $0,3 \mathrm{a}$ \\
8,0 & $0,0 \mathrm{a}$ & $0,2 \mathrm{a}$ \\
\hline C.V. $(\%)$ & 13,3 & 61,6
\end{tabular}

IR: Índice de Repelencia. Indica tratamiento Neutro si IR $=1$; Repelente si IR $=<1$ y Atrayente si IR $=>1$. C.V.: Coeficiente de variación. 
Tabla 5. Efecto antialimentario del aceite esencial de frutos de Schinus molle sobre Sitophilus zeamais. Table 5. Antifeeding effect of essential oil of Schinus molle fruits against Sitophilus zeamais.

\begin{tabular}{cc}
\hline Concentración & Índice Antialimentario (IA) ${ }^{1 *}$ \\
\hline 0,5 & \% \\
1,0 & $37,8 \mathrm{~b}$ \\
2,0 & $39,8 \mathrm{ab}$ \\
4,0 & $47,5 \mathrm{ab}$ \\
8,0 & $53,4 \mathrm{ab}$ \\
16,0 & $71,2 \mathrm{a}$ \\
C.V. & 22,3 \\
\hline * Tratamientos con igual letra en la columna no difieren estadísticamente, Tukey $(\mathrm{P}>0,05)$. \\
C.V.: Coeficiente de variación. \\
${ }^{1}$ Datos transformados previamente al análisis de varianza con la fórmula arcoseno $(\mathrm{x} / 100)^{0,5}$
\end{tabular}

\section{LITERATURA CITADA}

Abbott, W.S. 1925. A method for computing the effectiveness of an insecticide. J. Econ. Entomol. 18(2):265-267.

Abdel-Sattar, E., A. Zaitoun, M. Farag, S. Gayed, and F. Harraz. 2010. Chemical composition, insecticidal and insect repellent activity of Schinus molle L. leaf and fruit essential oils against Trogoderma granarium and Tribolium castaneum. Nat. Prod. Res. 24(3):226-235.

Adams, R.P. 2007. Identification of essential oil component by gas chromatography/mass spectrometry. 804 p. Academic Press, New York, USA.

Andrade, Y., G. Silva, N. Zapata, M. Tapia, J. Rodríguez, y A. Lagunes. 2009. Toxicidad de polvos de canelo (Drimys winteri J. R. et G. Forster) contra Sitophilus zeamais Motschulsky (Coleoptera: Curculionidae) en laboratorio. Agro-Ciencia 25(1):33-40.

Asawalam, E.F., S.O. Emosairue, and A. Hassanali. 2008. Contribution of different constituents to the toxicity of the essential oil constituents of Vernonia amygdalina (Compositae) and Xylopia aetiopica (Annonaceae) on maize weevil, Sitophilus zeamais Motschulsky (Coleoptera: Curculionidae). African Journal of Biotechnology 7(16):2957-2962.

Awoke, Y., G. Asayew, G. Melaku, M. Derbew, S. Kedir, and N. Raja. 2014. Evaluation of certain plant leaf powders and aqueous extracts against maize weevil, Sitophilus zeamais Motsch. (Coleoptera: Curculionidae). Asian Journal of Agricultural Sciences 6(3):83-88.

Benzi, V., N. Stefanazzi, and A. Ferrero. 2009. Biological activity of essential oils from leaves and fruits of pepper tree (Schinus molle L.) to control rice weevil (Sitophilus oryzae L.). Chilean J. Agric. Res. 69(2):154-159.
Betancur, J., G. Silva, J. Rodríguez, S. Fisher, and N. Zapata. 2010. Insecticidal activity of Peumus boldus Molina essential oil against Sitophilus zeamais Motschulsky. Chilean J. Agric. Res. 70(3):399-407.

Bustos, G., F. Osses, G. Silva, M. Tapia, R. Hepp, and J. Rodríguez. 2009. Insecticidal properties of Peumus boldus Molina powder used alone and mixed with lime against Sitophilus zeamais Motschulsky (Coleoptera: Curculionidae). Chilean J. Agric. Res. 69(3):350-355.

Conti, B., A. Canale, P. Cioni, and G. Flamini. 2010. Repellence of essential oils from tropical and Mediterranean Lamiaceae against Sitophilus zeamais. Bulletin of Insectology 63:197-202.

Chirino, M., M. Cariac, y A. Ferrero. 2001. Actividad insecticida de extractos crudos de drupas de Schinus molle L. (Anarcadiaceae) sobre larvas neonatas de Cydia pomonella L. (Lepidoptera: Tortricidae). Bol. San. Veg. Plagas 27(3):305-314.

Descamps, L., N. Stefanazzi, C. Sanchez Chopa, y A. Ferrero. 2008. Actividad biológica de extractos vegetales de Schinus molle var. areira (Anacardiaceae) en Tribolium castaneum Herbst. (Insecta, Coleoptera, Tenebrionidae), plaga de grano almacenado. Bol. San. Veg. Plagas 34(4):595-605.

Farrar, R.R., J.D. Barbour, and G.C. Kennedy. 1989. Quantifying food consumption and growth in insects. Ann. Entomol. Soc. Am. 82(5):593-598.

Fernandes, E.T. and S. Favero. 2014. Óleo essensial de Schinus molle L. para o controle de Sitophilus zeamais Most. 1855 (Coleoptera: Curculionidae) em milho. Rev. Bras. de Agroecología 9(1):225-231.

Finney, D. 1971. Probit Analysis. 272 p. Cambridge University Press, Cambridge, London, UK. 
García-Lara, S., C. Espinosa, y D. Bergvinson. 2007. Manual de plagas en granos almacenados y tecnologías alternas para su manejo y control. Centro Internacional de Mejoramiento de Maíz y Trigo (CIMMYT), México D.F., México.

García-Lara, S., y D. Bergvinson. 2007. Programa integral para reducir pérdidas poscosecha en maíz. Agric. Téc. Méx. 33(2):181-189.

Halstead, D. 1963. External sex differences in stored-products Coleoptera. Bull. Entomol. Res. 54(1):119-134.

Hayouni, E., I. Chraief, M. Abedrabba, M. Bouix, J. Leveau, H. Mohammed and M. Hamdi. 2008. Tunisian Salvia officinalis L. and Schinus molle L. essential oils: their chemical compositions and their preservative effects against Salmonella inoculated in minced beef meat. Int. J. Food Microbiol. 125(3):242-251.

Huang, Y., S.H. Ho, H.C. Lee, and Y.L. Yap. 2002. Insecticidal properties of eugenol, isougenol and methyleugenol and their effects on nutrition of Sitophilus zeamais Mostsch. (Coleoptera: Curculionidae) and Tribolium castaeum (Herbst) (Coleoptera: Tenebrionidae). J. Stored Prod. Res. 38(5):403412.

Iannacone, J., y L. Alvariño. 2010. Toxicidad de Schinus molle L. (Ancardiaceae) en cuatro controladores biológicos de plagas agrícolas en el Perú. Acta Zoológica Mexicana 26(3):603-615.

Isman, M., C.M. Machial, S. Miresmaili, and L.D. Bainard. 2007. Essential oils-based pesticides: new insights from old chemistry. p. 201-209. In H. Ohkawa, H. Miyagawa and P.W. Lee (eds.) Pesticide chemistry. Crop protection, Public Health, Environmental Safety. WileyVCH Verlag Gmbh \& Co. KGaA, Wainheim, Germany.

Isman, M., J. Wilson, and R. Bradbury. 2008. Insecticidal activities of commercial rosemary oils (Rosmarinus officinalis) against larvae of Pseudaletia unipuncta and Trichoplusia ni in relation to their chemical compositions. Pharm. Biol. 46(1-2):82-87.

Kordali, S., M. Kesdek, and A. Cakir. 2007. Toxicity of monoterpenes against larvae and adults of Colorado potato beetle, Leptinotarsa decemlineata Say (Coleoptera: Chrysomelidae). Ind. Crops Prod. 26(3):278297.
Lagunes, A. 1993. Uso de extractos y polvos vegetales y polvos minerales para el combate de plagas del maíz y del frijol en la agricultura de subsistencia: memoria. Colegio de Postgraduados en Ciencias Agrícolas / Agencia para el Desarrollo Internacional de los Estados Unidos / Consejo Nacional de Ciencia y Tecnología de México / Bodegas Rurales CONASUPO, Montecillo, México.

Maganga, M., G. Gries, and R. Gries. 1996. Repellency of various oils and pine oil constituents to house flies (Diptera: Muscidae). Envirom. Entomol. 25(5):11821187.

Mazzonetto, F., y J. Vendramim. 2003. Efeito de pós de origem vegetal sobre Acanthoscelides obtectus (Say) (Coleoptera: Bruchidae) em feijão armazenado. Neotrop. Entomol. 32(1):145-149.

Murray, A., M. Frontera, M. Tomas, and M. Mulet. 2005. Gas chromatography-mass spectrometry study of the essential oils of Schinus longifolia (Lindl.) Speg., Schinus fasciculata (Griseb.) I.M. Johnst., and Schinus areira L. Z. Naturforsch. 60(1-2):25-29.

Nerio, L., S. Olivero-Verbel, and J. Stashenko. 2009. Repelent activity of essential oils from seven aromatics plants grown in Colombia against Sitophilus zeamais Motschulsky (Coleoptera). Journal of Stored Product Research 45:212-214.

Obeng-Ofori, D., and C. Reichmuth. 1997. Bioactivity of eugenol, a major component of essential oil of Ocimum suave (Wild.) against four species of stored-product Coleoptera. Int. J. Pest Manag. 43(1):89-94.

Obeng-Ofori, D., C. Reichmuth, A. Bekele, and A. Hassanali. 1998. Toxicity and protectant potential of camphor, a major component of essential oil of Ocimum kilimandscharicum, against four stored product beetles. Int. J. Pest Manag. 44(4):203-209.

Pérez, F., G. Silva, M. Tapia, y R. Hepp. 2007. Variación anual de las propiedades insecticidas de Peumus boldus sobre Sitophilus zeamais. Pesq. Agropec. Bras. 42(5):633-639.

Rodríguez, R., E. Ruiz., y J. Elissetche. 2005. Árboles en Chile. Universidad de Concepción, Concepción, Chile.

Salvadores, Y., G. Silva, M. Tapia, y R. Hepp. 2007. Polvos de especies aromáticas para el control del gorgojo del maíz, Sitophilus zeamais Motschulsky, en trigo almacenado. Agric. Téc. (Chile) 67(2):147-154. 
SAG. 2000. Normas generales y específicas de certificación de semillas. Ministerio de Agricultura de Chile. Servicio Agrícola y Ganadero. Departamento de Semillas. Santiago. Chile. Disponible en http://www. sag.gob.cl/sites/default/files/NORMAS GENERALES_SEMILLAS.pdf. (Consultado diciembre 2015).

Silva, G., A. Lagunes, y J. Rodríguez. 2003. Control de Sitophilus zeamais (Coleóptera: Curculionidae) con polvos vegetales solos y en mezcla con carbonato de calcio en maíz almacenado. Cien. Investig. Agrar. 30(3):153160.

Silva, G., O. Orrego, R. Hepp, y M. Tapia. 2005. Búsqueda de plantas con propiedades insecticidas para el control de Sitophilus zeamais en maíz almacenado. Pesq. Agropec. Bras. (Brasilia) 40(1):11-17.

Tavares, M., e D. Vendramim. 2005. Bioatividade da Erva-de-Santa-Maria, Chenopodium ambrosioides L., sobre Sitophilus zeamais Mots. (Coleoptera: Curculionidae). Neotrop. Entomol. 34(2):319-323.

Tefera, T., S. Mugo, and P. Likhayo. 2011. Effects of insect population density and storage time on grain damage weight loss in maize due to the maize weevil Sitophilus zeamais and the larger grain borer Prostephanus truncatus. African Journal of Agricultural Research 6:2249-2254.
Tripathi, A., S. Upadhyay, M. Bhuiyan, and P.R. Brattacharya. 2009. A review on prospects of essential oils as biopesticide in insect-pest management. Journal of Pharmacognosy and Phytotherapy 1(5):52-63.

Vogel, H., I. Razmilic, y U. Doll. 1997. Contenido de aceite esencial y alcaloides en diferentes poblaciones de boldo (Peumus boldus Mol.). Cien. Investig. Agrar. 24(1):1-6.

Werdin, J., A. Murray, y A. Ferrero. 2008. Bioactividad de aceites esenciales de Schinus molle var. areira (Anacardiaceae) en ninfas II de Nezara viridula (Hemiptera: Pentatomidae). Bol. Sanid. Veg., Plagas 34(3):367-375.

Wimalaratne, P.D.C., K.N. Slessor, J.H. Borden, L.J. Chong, and T. Abate. 1996. Isolation and identification of house fly, Musca domestica L., repellents from pepper tree, Schinus molle L. J. Chem. Ecol. 22(1):49-50. 